\title{
The Quantum Properties of a Superposition of Vee Type Three-level Lasers
}

\author{
Takele Teshome \\ Department of Physics, College of Natural and Computational Sciences, Wolaita Sodo, Ethiopia
}

Email address:

takeleteshome36@gmail.com

\section{To cite this article:}

Takele Teshome. The Quantum Properties of a Superposition of Vee Type Three-level Lasers. American Journal of Physics and Applications. Vol. 9, No. 4, 2021, pp. 64-69. doi: 10.11648/j.ajpa.20210904.11

Received: May 26, 2021; Accepted: July 13, 2021; Published: July 22, 2021

\begin{abstract}
The squeezing, and statistical properties of a superposed light beam produced by a lambda type three-level lasers configuration have been studied. We have determined the quadrature variances mean as well as variance photon number for cavity modes with the aid of the solutions of c-number Langevin equations associated with the normal order. We have carried out our analysis a light in a squeezing state can be produced by the system under consideration under the condition that the cavity decay constant is larger than the linear gain coefficient and the squeezing occurs in the minus-quadrature. Furthermore, we also obtain with the aid of the Q-functions and the density operator the superposition beam, and superposed light beams are determined in quadrature variance and mean photon number. The result shows that the mean photon number and the quadrature variance of the superposed light beam are the sum of the mean photon number and the quadrature variance of the constituent light beams.
\end{abstract}

Keywords: Laser, Quadrature Squeezing, Photon Statistics

\section{Introduction}

Quantum optics deals mainly with the quantum properties of light generated by various optical systems such as lasers with the effects of light on the dynamics of the atoms. The interaction between radiation and matter (atoms) lies at the heart of quantum optics. There has been a considerable interest in the analysis of squeezing and statistical properties of the light generated by three-level lasers $[1,2,14]$. A threelevel laser may be defined as a quantum optical system in which three-level atoms initially prepared in a coherent superposition of the two levels, are injected into a cavity coupled to a vacuum reservoir via a single-port mirror. Three-level system involves two dipole transients. The three types of three-level systems, all taking their names from Greek and Latin letter are Lambda $\Lambda$ system which have two lower levels coupled to an excited one, Cascade $\Xi$ system which have an excited state, coupled to a middle state, and the middle one coupled to the ground state, and $V_{\text {ee }}(\mathrm{V})$ system, which have one ground state coupled to two excited states. A three-level laser consists of a cavity into which three-level atoms are injected at a constant rate and removed from the cavity after a certain time $\tau \mathrm{T}$. The three levels are represented by $|a>| b>$,, and $\mid c>[1,4,5,8]$.

Squeezing is one of the non-classical features of light that has attracted a great deal of interest. In squeezed light, the noise in one quadrature is below the coherent or vacuum state level at the expense of enhanced fluctuations in the other quadrature with the product of the uncertainty in the two quadrature satisfying the uncertainty relation. [1,3] Squeezed light has potential application in low noise optical communication and weak signal detection. A three-level laser may be defined as a quantum optical system in which threelevel atoms, initially prepared in a coherent superposition of the two levels, are injected into a cavity. When a three-level atom makes a transition from top to the bottom levels, two photons are generated. The coupling of the two levels of the atom is responsible for the interesting non classical features of the generated light. In general, the atomic coherence can be induced in a three-level atom by coupling with coherent light or by preparing the atom's initially incoherent superposition of these two levels. There are several quantum optical systems that could generate light with non-classical features such as squeezing, entanglement, and anti-bunching etc. $[10,15,17]$.

We assume the cavity modes to be at resonance with the 
two transition $|\mathrm{a}>\leftrightarrow| \mathrm{b}>$ and $|\mathrm{a}>\leftrightarrow| \mathrm{b}>$ are dipole allowed with direct transition between level $|a>\rightarrow| c>$ to be dipole forbidden. It is considered that the case in which the atoms are initially prepared in Superposition of $\mid a>$ and $|c\rangle$. When the three-level cascade atom decays from upper to the lower levels two photons are emitted $[6,7,9,16]$. If the two photons have identical frequencies, then the three-level atom is referred to as a degenerate three-level atom, otherwise it is called non degenerate three-level atom [11-13]. It is realized that the two photons generated by a cascade three-level laser system have squeezing properties under certain conditions due to the correlation between the photons.

\section{Three-Level Atom with Radiation}

In this chapter we seek to drive the Hamiltonian describing the interaction of a radiation with Vee type three-level atom and the master equation for three-level laser coupled to vacuum reservoir.

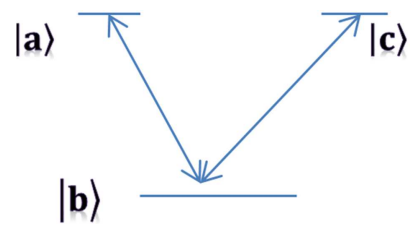

Figure 1. Schematic representation of a three-level atom in a Vee Configuration.

\subsection{The Hamiltonian}

In this section we seek to drive the Hamiltonian describing the interaction of a radiation with three-level atom. The interaction of a one-electron atom having mass $m$ and charge e with a single-mode radiation represented by the vector potential $A$, is described by the Hamiltonian.

$$
\widehat{H}_{1}=\frac{e}{m} \hat{p} \cdot \hat{A}+\frac{e^{2}}{2 m} \hat{A}^{2}
$$

Where $\hat{P}$ is the canonical momentum? Since, we can neglect the second term in Eqn. (1). Hence the interaction Hamiltonian can be put in the form

$$
\widehat{H}_{1}=\frac{e}{m} \hat{p} \cdot \hat{A}
$$

It proves to be more convenient to express this Hamiltonian in terms of the electric field operator. To this end, we note that

$$
\frac{d}{d t}(\hat{r} . \hat{A})=-\frac{i e}{m \hbar}[\hat{r} \cdot \hat{A}, \hat{p} . \hat{A}]
$$

$\mathrm{n}$ which $\hat{r}$ is the position operator for the electron. Taking into account the fact that the vector potential commute with the position and momentum operators, one readily finds

$$
[\hat{r} . \hat{A}, \hat{p} . \hat{A}]=i \hbar \hat{A}^{2} .
$$

It then follows that

$$
\frac{d}{d t}(\hat{r} . \hat{A})=\frac{e}{m} \hat{A}^{2}
$$

On the other hand, we have

$$
\frac{d}{d t}(\hat{r} \cdot \hat{A})=\hat{A} \cdot \frac{d \hat{r}}{d t}+\hat{r} \cdot \frac{d \hat{A}}{d t},
$$

So that combination of Eqn. (5) and (6) leads to

$$
\hat{A} \cdot \frac{d \hat{r}}{d t}=\frac{e}{m} \hat{A}^{2}-\hat{r} \cdot \frac{d \hat{A}}{d t}
$$

Furthermore, with the aid of the relation

$$
\hat{p}=\mathrm{m} \frac{d \hat{r}}{d t}-\mathrm{e} \hat{A}
$$

One can write

$$
\hat{p} \cdot \hat{A}=\mathrm{m} \mathrm{d} \frac{d \hat{r}}{d t} \cdot \hat{A}-\mathrm{e} \hat{A}^{2}
$$

Now on account of Eqn. (7) and (9), we have

$$
\hat{p} \cdot \hat{A}=-\mathrm{m} \hat{r} \cdot \frac{d \hat{A}}{d t} .
$$

In view of this the interaction is the Hamiltonian described by Eqn. (2) can therefore be

Put in the form

$$
\widehat{H}_{\mathrm{I}}=-\mathrm{e} \hat{r} \cdot \frac{d \hat{A}}{d t}
$$

It can be easily established that

$$
\frac{d \hat{A}}{d t}=\frac{\partial \hat{A}}{\partial t}+(-\vec{v} \cdot \nabla) \hat{A}
$$

A simpler form of Eqn. (12) can be obtained by applying the electric dipole approximation

$$
e^{i k \cdot r}=1
$$

This is evidently justified and provided that $\mathrm{k} . \mathrm{r}<<1$. Inspection of the vector potential in the electric dipole approximation, on the position coordinates, it then turns out in this approximation that

$$
(-\vec{v} \cdot \nabla) \hat{A}=0,
$$

And hence in the Coulomb gauge we have

$$
\frac{d \hat{A}}{d t}=\frac{\partial \hat{A}}{\partial t}=-\hat{E}
$$

In view of this result, we see that

$$
\widehat{H}_{\mathrm{I}}=\mathrm{e} \hat{r}(\mathrm{t}) \cdot \hat{E}(\mathrm{x}, \mathrm{t}) \text {. }
$$

\subsection{The Quantum Approximation}

In this section we approximate the operators in mathematically expressed. The electric field operator for two-mode light in the electric dipole approximation has the form 


$$
\hat{E}(x, t)=\mathrm{ie}\left\{\left[\frac{\hbar \omega_{a}}{2 E_{o V}}\right]^{\frac{1}{2}}\left(\hat{a} e^{-i \omega_{a} t}-\hat{a}^{+} e^{i \omega_{a} t}\right)+\left\{\left[\frac{\hbar \omega_{b}}{2 E_{o V}}\right]^{\frac{1}{2}}\left(\hat{b} e^{-i \omega_{b} t}-\hat{b}^{+} e^{i \omega_{b} t}\right)\right\} \mathrm{u}\right.
$$

And

$$
\hat{r}(t)=r_{a b}\left(\hat{\sigma}_{a b} e^{i \omega_{a}^{\prime} t}+\hat{\sigma}_{b a} e^{-i \omega_{a}^{\prime} t}\right)+r_{b c}\left(\hat{\sigma}_{b c} e^{i \omega_{b}^{\prime} t}+\hat{\sigma}_{c b} e^{-i \omega_{b}^{\prime} t}\right)
$$

The result is

$$
\widehat{H} \mathrm{I}=\mathrm{i} \hbar g\left[\left(\hat{\sigma}_{a b} \hat{a} e^{-i\left(\omega_{a}-\omega_{a}^{\prime}\right) t}-\hat{\sigma}_{b a \hat{a}^{+}} e^{-i\left(\omega_{a}-\omega_{a}^{\prime}\right) t}\right)+\left(\hat{\sigma}_{b c \hat{b}} e^{i\left(\omega_{b}-\omega_{b}^{\prime}\right) t}+\hat{\sigma}_{c b} \hat{b}^{+} e^{-i\left(\omega_{b}-\omega_{b}^{\prime}\right) t}\right)\right] .
$$

\section{Master Equation}

In this section we determine the master equation for Vee type three-level laser in two-mode vacuum reservoir

\subsection{Vee- Type Three-Level Laser}

We consider three-level lasers in which three-level atoms in $\mathrm{V}$ configuration are injected at a constant rate ra and removed from the cavity after a certain time $\tau$. We denote the top and the two bottom levels of three-level atom by $|\mathrm{b}>,| \mathrm{a}>$ and $\mid \mathrm{c}>$ as shown in Figure 1. We assume that the cavity modes to be at resonance with the two transitions $|\mathrm{b}>\leftrightarrow| \mathrm{a}>$ and $|\mathrm{b}>\leftrightarrow| \mathrm{c}>$, are dipole allowed and direct transition between level $|\mathrm{a}>\leftrightarrow| \mathrm{c}>$ to be dipole forbidden. The interaction of $\mathrm{V}$ three-level atom with the cavity modes can be described in the interaction picture by the Hamiltonian

$$
\widehat{H}_{\mathrm{I}}=\mathrm{i} g\left[\left(\hat{\sigma}_{a b} \hat{a}-\hat{\sigma}_{b a \hat{a}^{+}}\right)+\left(\hat{\sigma}_{b c \hat{b}}+\hat{\sigma}_{c b} \hat{b}^{+}\right)\right]
$$

We take the initial state of a three-level atom to be

$$
\psi_{A}(0)=C a(0)|a>+C c(0)| c>
$$

The master equation for $\mathrm{V}$ three-level laser coupled to a two-mode vacuum reservoir can be

Written as

$$
\begin{aligned}
\frac{d}{d t} \hat{\rho} & =\frac{1}{2}\left(\kappa+B \rho_{a a}^{(0)}\right)\left(2 \hat{a} \hat{\rho} \hat{a}^{+}-\hat{a}^{+} \hat{a} \hat{\rho}-\hat{\rho} \hat{a}^{+} \hat{a}\right)+\frac{1}{2}\left(\kappa+B \rho_{c c}^{(0)}\right)\left(2 \hat{b} \hat{\rho} \hat{b}^{+}-\hat{b}+\hat{b} \hat{\rho}-\hat{\rho} \hat{b}^{+} \hat{b}\right) \\
& +\frac{1}{2}\left(B \rho_{a c}^{(0)}\right)\left(2 \hat{a} \hat{\rho} \hat{b}^{+}-\hat{\rho} \hat{b}^{+} \hat{a}-\hat{b}^{+} \hat{a} \hat{\rho}\right)+\frac{1}{2}\left(B \rho_{a c}^{(0)}\right)\left(2 \hat{b} \hat{\rho} \hat{a}^{+}-\hat{a}^{+} \hat{b} \hat{\rho}-\hat{\rho} \hat{a}^{+} \hat{b}\right)
\end{aligned}
$$

\subsection{C-Number Langevin Equations}

The c- number Langevin equation associated with the normal orders.

$$
\begin{aligned}
& \frac{d}{d t}\left\langle\alpha>=-\frac{1}{2} \mu_{a}\left\langle\alpha>-\frac{1}{2} v<\beta>\right.\right. \\
& \frac{d}{d t}<\beta>=-\frac{1}{2} \mu_{c}<\beta>-\frac{1}{2} v<\alpha> \\
& \begin{array}{l}
\frac{d}{d t}\left\langle\alpha^{*} \beta\right\rangle=-\frac{1}{2}\left(\mu_{a}+\mu_{c}\right) \\
\qquad \frac{d}{d t}\langle\alpha \beta\rangle=-\frac{1}{2}\left(\mu_{a}+\mu_{c}\right) \\
\text { tion Eqns. (29) and (30] } \\
\frac{1}{2} \mu_{a} \alpha(t)-\frac{1}{2} v \beta(t)+f_{\alpha}(t)
\end{array} \\
& \frac{d}{d t} \alpha(t)=-\frac{1}{2} \mu_{a} \alpha(t)-\frac{1}{2} v \beta(t)+f_{\alpha}(t) \\
& \frac{d}{d t} \beta(t)=-\frac{1}{2} \mu_{c} \beta(t)-\frac{1}{2} v \alpha(t)+f_{\beta}(t)
\end{aligned}
$$

$$
\frac{d}{d t}<\alpha^{2}>=-\mu_{a}<\alpha^{2}>-v<\alpha \beta>
$$$$
\frac{d}{d t}<\beta^{2}>=-\mu_{c}<\beta^{2}>+v<\alpha \beta>
$$

Where $f_{\alpha}(t)$ and $f_{\beta}(t)$ are noise forces. The solution of equation (31) and (32) can be written as

$$
\begin{aligned}
& \alpha(t)=\alpha(0) e^{\frac{-\mu_{a} t}{2}}-\int_{0}^{t} d t^{\prime} e^{\frac{-\mu_{a}\left(t-t^{\prime}\right)}{2}}\left(\frac{1}{2} v \beta\left(t^{\prime}\right)-f_{\beta}\left(t^{\prime}\right)\right) \\
& \beta(t)=\beta(0) e^{\frac{-\mu_{c} t}{2}}-\int_{0}^{t} d t^{\prime} e^{\frac{-\mu_{c}\left(t-t^{\prime}\right)}{2}}\left(\frac{1}{2} v \alpha\left(t^{\prime}\right)-f_{\beta}\left(t^{\prime}\right)\right)
\end{aligned}
$$

The solution of coupled differential equations Eqn. (33)

$$
\begin{gathered}
\frac{d}{d t}\left\langle\alpha^{*} \alpha>=-\mu_{a}<\alpha^{*} \alpha>-\frac{1}{2} v\left(<\alpha^{*} \beta>+<\beta^{*} \alpha>\right)\right. \\
\frac{d}{d t}\left\langle\beta^{*} \beta>=-\mu_{c}<\beta^{*} \beta>-\frac{1}{2}\left(\mu<\alpha^{*} \beta>+v \beta^{*} \alpha>\right)\right.
\end{gathered}
$$

and Eqn. (34) can be written in the matric form as

$$
\begin{aligned}
\frac{d}{d t} Y(t) & =\frac{1}{2} M Y(t)+F(t) \\
Y(t) & =(\alpha(t), \beta(t)) \\
M & =\left(\mu_{a} v v \mu_{c}\right) \\
F(t) & =\left(f_{\alpha}(t) f_{\beta}(t)\right)
\end{aligned}
$$

We next proceed to find the eigenvalues and eigenvectors of the matrix M. Applying the eigenvalue equation $M U_{i}=\lambda U_{i}$, We find the characteristic equation

$$
\lambda^{2}-\lambda\left(\mu_{a}+\mu_{c}\right)+\mu_{c} \mu_{a}-v^{2}=0
$$

We finally obtain 


$$
\begin{aligned}
& \alpha(t)=p_{1}(t) \alpha(0)+q_{1}(t) \beta(0)+H_{1}(t) \\
& \beta(t)=p_{2}(t) \beta(0)+q_{2}(t) \alpha(0)+H_{2}(t)
\end{aligned}
$$

Where

$$
\begin{aligned}
& H_{1}(t)=\int_{0}^{t} p_{1}\left(t-t^{\prime}\right) f_{\alpha}\left(t^{\prime}\right)+q_{1}\left(t-t^{\prime}\right) f_{\beta}\left(t^{\prime}\right) d t^{\prime} \\
& H_{2}(t)=\int_{0}^{t} p_{2}\left(t-t^{\prime}\right) f_{\beta}\left(t^{\prime}\right)+q_{2}\left(t-t^{\prime}\right) f_{\alpha}\left(t^{\prime}\right) d t^{\prime}
\end{aligned}
$$

\subsection{The $Q-F u n c t i o n$}

The cavity mode produced by a Vee three level laser coupled to a vacuum reservoir. The $\mathrm{Q}$ function for a twomode light is expressible as

$$
Q(\alpha, \beta, t)=\frac{1}{\pi^{2}} \int \frac{d^{2} z}{\pi} \frac{d^{2} w}{\pi} \Phi(z, w, t) e^{z * \alpha-z \alpha *+w * \beta-w \beta *}
$$

Where

$$
\Phi(\mathrm{z}, \mathrm{w}, \mathrm{t})=\operatorname{Tr}\left(\hat{\rho} e^{-z * \hat{a}(t)} e^{z \hat{a}^{+}(t)} e^{-w * \hat{b}(t)} e^{w \hat{b}^{+}(t)}\right.
$$

We can write in terms of c-number variables associated to the normal ordering as

$$
\Phi(\mathrm{z}, \mathrm{w}, \mathrm{t})=e^{-z * z} e^{-w * w}<e^{z \alpha *(t)-z * \alpha(t)+w \beta *(t)-w * \beta(t)}>
$$

We note that $\alpha(t)$ and $\beta(t)$ are Gaussian variables with a vanishing mean.

In the view of this finally Q-function for Vee type threelevel lasers,

$$
\Delta c^{2}{ }_{ \pm}=4+2<\hat{c}^{+} \hat{c}> \pm<\hat{c}^{+2}> \pm<\hat{c}^{2}>-2<\hat{c}^{+}><\hat{c}>\mp<\hat{c}^{+}>^{2} \mp<\hat{c}>^{2} .
$$

Taking to account Eqn. (50) and $<\hat{c}^{+}>=<\hat{c}>$, we have

$$
\Delta c^{2}{ }_{ \pm}=4+2<\hat{c}^{+} \hat{c}> \pm 2<\hat{c}^{2}>
$$

Using the density operator, we write as and c- Langevin equation

$$
\Delta c^{2}{ }_{ \pm}=4+A(1-\eta)\left[\frac{2 \kappa(4 \kappa+2 A \eta+A)-A^{2}(1-\eta)}{2 \kappa(2 \kappa=A \eta)(\kappa+A \eta)}\right]+A \sqrt{1-\eta^{2}}\left[\frac{2 \kappa(4 \kappa+3 A \eta+A)-A^{2}\left(1-\eta^{2}\right)}{2 \kappa(2 \kappa=A \eta)(\kappa+A \eta)}\right]
$$

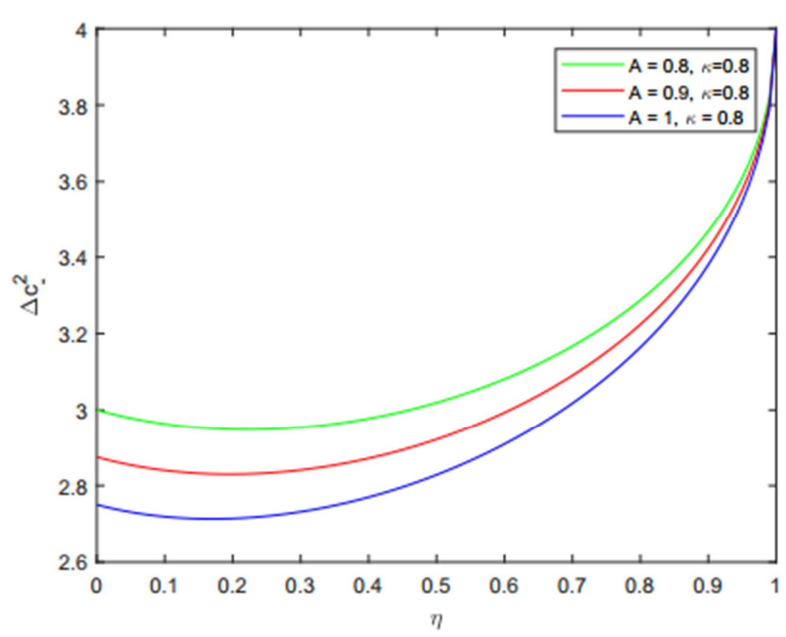

Figure 2. Plots of the minus quadrature variance above equation versus $\eta$ for $\kappa=0.8$, and for different values of the line again coefficient.
The equation represents the quadrature variances for superposition of the light beams produced by Vee three-level lasers. Figure 2 represents the variances of the minus quadrature [Eqn. (52)] versus $\eta$ for different values of $A$. This figure indicates that the degree of squeezing increases with the linear gain coefficient and almost perfect squeezing can be obtained for large values of the linear gain coefficient and for small values of $\eta$. Moreover, the minimum value of the quadrature variance described by Eqn. (53) for $A=1, \kappa=0.8$, is found to be $\Delta c_{\overline{2}}^{-}=2.79$ and occurs at $\eta=0.35$ this result implies that the maximum intracavity squeezing for the above values is 30.25 percent below the coherent-state level.

\section{Photon Statistics}

In this chapter we wish to study the statistical properties of a superposition of a two-mode light beam produced by Vee-type three- level lasers employing the individual beams' Q- 
functions and the density operator for the superposed beams. We consider the two light beams from perpendicular direction propagating into a mirror having one side totally transmitting and the other side totally reflective as seen in Figure 3.

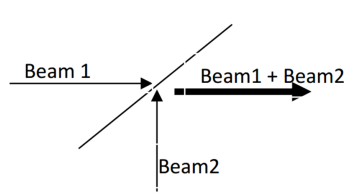

Figure 3. Schematic representation of the superposition of two-mode light beams of lambda-type three-level lasers.

\subsection{Density Operator}

Suppose $\curvearrowright \rho(t)$ be the density operator for the superposition of two beams. We can write $\hat{\rho}(\mathrm{t})$ in the normal order as

$$
\begin{gathered}
\hat{\rho}(t)=\sum_{i j k l} c_{i j k l}(t) \hat{a}^{+i} \hat{a}^{j} \hat{b}^{+k} \hat{a}^{l} \\
\hat{I}=\int \frac{d^{2} \alpha_{1}}{\pi} \frac{d^{2} \beta_{1}}{\pi}\left|\alpha_{1} \beta_{1}\right\rangle\left\langle\beta_{1} \alpha_{1}\right|, \text { and the relation } \\
4\left|\alpha_{1} \beta_{1}\right\rangle\left\langle\beta_{1} \alpha_{1}\left|\hat{a}^{+i} \hat{b}^{+k}=\hat{a}^{+i} \hat{b}^{+k}\right| \alpha_{1} \beta_{1}\right\rangle\left\langle\beta_{1} \alpha_{1}\right|,
\end{gathered}
$$

Where

$$
\begin{aligned}
Q_{1}=\left(\alpha_{1}^{*}, \beta_{1}^{*}, \alpha_{1}+\frac{\partial}{\partial \alpha_{1}^{*}} ; \beta_{1}+\frac{\partial}{\partial \beta_{1}^{*}}\right) & =\frac{1}{2 \pi} \sum_{i j k l} c_{i j k l}(t) \alpha_{1}^{* i} \beta_{1}^{* l}\left(\alpha_{1}+\frac{\partial}{\partial \alpha_{1}^{*}}\right)^{j}\left(\beta_{1}+\frac{\partial}{\partial \beta_{1}^{*}}\right)^{k} \\
\widehat{D}\left(\alpha_{1}, \beta_{1}\right) & =e^{\alpha_{1} \hat{a}^{+}+\beta_{1} \hat{b}^{+}-\alpha_{1}^{*} \hat{a}-\beta_{1}^{*} \hat{b}}
\end{aligned}
$$

And $\hat{\rho}_{o}$ is the density operator for the system at initial time

Assuming that the density operator at initial time is to be in some other state and following the same procedure to obtain the expression in Eqn. (58), we readily find that

$$
\hat{\rho}_{o}=\int d^{2} \alpha_{2} d^{2} \beta_{2} Q_{2}\left(\alpha_{2}^{*}, \beta_{2}^{*}, \alpha_{2}+\frac{\partial}{\partial \alpha_{2}^{*}} ; \beta_{2}+\frac{\partial}{\partial \beta_{2}^{*}}\right) \widehat{D}\left(\alpha_{2}, \beta_{2}\right)|0,0\rangle\langle 0,0| \widehat{D}^{+}\left(\alpha_{2}, \beta_{2}\right)
$$

In which

$$
\begin{gathered}
Q_{2}=\left(\alpha_{2}^{*}, \beta_{2}^{*}, \alpha_{2}+\frac{\partial}{\partial \alpha_{2}^{*}} ; \beta_{2}+\frac{\partial}{\partial \beta_{2}^{*}}\right)=\frac{1}{2 \pi} \sum_{p q r s} c_{p q r s}(t) \alpha_{2}^{* p} \beta_{2}^{* r}\left(\alpha_{2}+\frac{\partial}{\partial \alpha_{2}^{*}}\right)^{q}\left(\beta_{2}+\frac{\partial}{\partial \beta_{2}^{*}}\right)^{s} \\
\widehat{D}\left(\alpha_{2}, \beta_{2}\right)=e^{\alpha_{2} \hat{a}^{+}+\beta_{2} \hat{b}^{+}-\alpha_{1}^{*} \hat{a}-\beta_{2}^{*} \hat{b}}
\end{gathered}
$$

With the aid of Eqns. (58) and (59) as well as the relation

$$
\widehat{D}\left(\alpha_{2}, \beta_{2}\right) \widehat{D}\left(\alpha_{1}, \beta_{1}\right)|0,0\rangle\left\langle 0,0\left|\widehat{D}^{+}\left(\alpha_{1}, \beta_{1}\right) \widehat{D}^{+}\left(\alpha_{2}, \beta_{2}\right)=\right| \alpha_{1}+\alpha_{2}+\beta_{1}+\beta_{2}\right\rangle\left\langle\beta_{1}+\beta_{2}+\alpha_{1}+\alpha_{2}\right|
$$

The expectation value of the annihilation operator for twomode superposed light beams represented by ${ }^{\wedge} c$ in terms of density operator is given by

Taking account Eqn. (61) and $\int d^{2} \alpha_{i} d^{2} \beta_{i} Q_{i}\left(\alpha_{i}^{*}, \beta_{i}^{*}, \alpha_{i}+\right.$ $\left.\frac{\partial}{\partial \alpha_{i}^{*}} ; \beta_{i}+\frac{\partial}{\partial \beta_{i}^{*}}\right)=1$, we have

$$
\begin{gathered}
\langle\hat{a}\rangle=\operatorname{Tr}(\hat{\rho}(t) \hat{c}(0)) \\
\langle\hat{c}\rangle=\left\langle\hat{a}_{1}\right\rangle+\left\langle\hat{b}_{1}\right\rangle+\left\langle\hat{a}_{2}\right\rangle+\left\langle\hat{b}_{2}\right\rangle \\
\left\langle\hat{a}_{i}\right\rangle=\int d^{2} \alpha_{i} d^{2} \beta_{i} Q_{i}\left(\alpha_{i}^{*}, \beta_{i}^{*}, \alpha_{i}+\frac{\partial}{\partial \alpha_{i}^{*}} ; \beta_{i}+\frac{\partial}{\partial \beta_{i}^{*}}\right) \alpha_{i} \\
\left\langle\hat{b}_{i}\right\rangle=\int d^{2} \alpha_{i} d^{2} \beta_{i} Q_{i}\left(\alpha_{i}^{*}, \beta_{i}^{*}, \alpha_{i}+\frac{\partial}{\partial \alpha_{i}^{*}} ; \beta_{i}+\frac{\partial}{\partial \beta_{i}^{*}}\right) \beta_{i}
\end{gathered}
$$

with $i=1,2$. Based on the result given by Eqn. (64), one can write the operator representing the superposed light beams as

$$
\hat{c}=\hat{a}_{1}+\hat{a}_{2}+\hat{b}_{1}+\hat{b}_{2}
$$

With the commutation relation

$$
\left[\hat{c}, \hat{c}^{+}\right]=4 .
$$

We note that the Q-function for a single mode can obtained using the relation

$$
\begin{gathered}
Q_{i}\left(\alpha_{i}^{*}, \alpha_{i}\right)==\int d^{2} \beta_{i} Q_{i}\left(\alpha_{i}, \beta_{i}, t\right) \\
Q_{i}\left(\alpha_{i}^{*}, \alpha_{i}+\frac{\partial}{\partial \alpha_{i}^{*}}\right)=Q_{i}\left(\alpha_{i}^{*}, \alpha_{i}, t\right) e^{-U a_{i} \alpha_{i}^{*} \frac{\partial}{\alpha_{i}^{*}}} \\
Q_{i}\left(\beta_{i}^{*},+\frac{\partial}{\partial \beta_{i}^{*}}\right) \beta_{i}=Q_{i}\left(\beta_{i}^{*}, \beta_{i}, t\right) e^{-U a_{i} \beta_{i}^{*} \frac{\partial}{\beta_{i}^{*}}}
\end{gathered}
$$

\subsection{Mean Photon Number}

In this section we seek to calculate the mean photon number for superposed two- mode light beams produced by Vee- type three -level lasers. The mean photon number for the superposition of two-mode light beams can be expressed 
in terms of the density operator as

$$
\underline{n}=\left\langle\hat{c}^{+} \hat{c}\right\rangle=\operatorname{Tr}\left(\hat{\rho} \hat{c}^{+} \hat{c}\right)
$$

$$
\hat{C}^{+}(t) \hat{c}(t)=\int d^{2} \alpha_{1} d^{2} \alpha_{2} d^{2} \beta_{1} d^{2} \beta_{2} Q_{1}\left(\alpha_{1}^{*}, \beta_{1}^{*}, \alpha_{1}+\frac{\partial}{\partial \alpha_{1}^{*}} ; \beta_{1}+\frac{\partial}{\partial \beta_{1}^{*}}\right)\left(\alpha_{2}^{*}, \beta_{2}^{*}, \alpha_{2}+\frac{\partial}{\partial \alpha_{2}^{*}} ; \beta_{2}+\frac{\partial}{\partial \beta_{2}^{*}}\right)\left|\alpha_{1}+\alpha_{2}+\beta_{1}+\beta_{2}\right|^{2}
$$

This equation can be put in the form

$$
\begin{gathered}
\hat{C}^{+}(t) \hat{c}(t)=\left\langle a_{1}^{+} \hat{a}_{1}\right\rangle+\left\langle a_{2}^{+} \hat{a}_{2}\right\rangle+\left\langle\hat{b}_{1}^{+} \hat{b}_{1}\right\rangle+\left\langle\hat{b}_{2}^{+} \hat{b}_{2}\right\rangle+\left\langle a_{1}^{+} \hat{b}_{1}\right\rangle+\left\langle\hat{b}_{1}^{+} \hat{a}_{1}\right\rangle+\left\langle\hat{b}_{2}^{+} \hat{a}_{2}\right\rangle+\left\langle\hat{a}_{2}^{+} \hat{b}_{2}\right\rangle+\left\langle\hat{b}_{2}^{+} \hat{a}_{2}\right\rangle+\left\langle\hat{a}_{1}^{+}\right\rangle\left\langle\hat{a}_{2}\right\rangle \\
+\left\langle\hat{a}_{2}^{+}\right\rangle\left\langle\hat{a}_{1}\right\rangle+\left\langle\hat{a}_{1}^{+}\right\rangle\left\langle\hat{b}_{2}\right\rangle+\left\langle\hat{b}_{2}^{+}\right\rangle\left\langle\hat{a}_{1}\right\rangle+\left\langle\hat{b}_{1}^{+}\right\rangle\left\langle\hat{a}_{2}\right\rangle+\left\langle\hat{a}_{2}^{+}\right\rangle\left\langle\hat{b}_{1}\right\rangle+\left\langle\hat{b}_{1}^{+}\right\rangle\left\langle\hat{b}_{2}\right\rangle+\left\langle\hat{b}_{2}^{+}\right\rangle\left\langle\hat{b}_{1}\right\rangle
\end{gathered}
$$

Carrying out the integration and then performing the differentiation, we find

$$
\begin{gathered}
\left\langle\hat{a}_{i}^{+} \hat{a}_{i}\right\rangle=a_{1}-1 \\
\left\langle a_{1}^{+} \hat{a}_{1}\right\rangle=a_{1}-1\left\langle a_{2}^{+} \hat{a}_{2}\right\rangle=0
\end{gathered}
$$

Similarly

$$
\begin{gathered}
\left\langle\hat{b}_{i}^{+} \hat{b}_{i}\right\rangle=b_{1}-1 \\
\left\langle\hat{b}_{1}^{+} \hat{b}_{1}\right\rangle=b_{1}-1,\left\langle\hat{b}_{2}^{+} \hat{b}_{2}\right\rangle=o \\
\left\langle\hat{a}_{i}\right\rangle=\left\langle\hat{b}_{i}\right\rangle=\left\langle\hat{a}_{i}^{+} \hat{b}_{i}\right\rangle=0
\end{gathered}
$$

Then finally

$$
\hat{C}^{+}(t) \hat{c}(t)=\sum_{1}^{2}\left\langle\hat{a}_{i}^{+} \hat{a}_{i}\right\rangle+\left\langle\hat{b}_{i}^{+} \hat{b}_{i}\right\rangle
$$

Eqn. (78) represents the mean photon number for the superposition of the cascade and lambda three-level lasers. We observe from Eqn. (78) that the mean photon number for superposition light beams is the sum of the mean photon number of the mean photon number of a lambda -type three-level laser.

\section{Conclusion}

In this research we have seen the squeezing and statistical properties of the light generated by three- level lasers whose cavity modes are coupled to vacuum reservoirs. In which the three-level atoms in a Vee configuration and initially prepared in the superposition of the top and bottom levels are injected into a cavity coupled to vacuum reservoir via a single port-mirror. Applying the linear and adiabatic approximation scheme we found the master equation for a light produced by three -level lasers from which we obtained the $\mathrm{c}$-number Langevin equations and their solutions. Employing these solutions we found the ant normally ordered characteristic function which was used to find the Q-function of a light beam generated by three -level lasers in Vee.

Upon applying the Q-function we calculated the mean photon number and the quadrature variance Moreover; we have calculated the Q-function for the superposition of Veetype three level laser light beams. Applying this function we calculated the density operator, the mean photon number and quadrature variance for steady state. We have seen that the degree of squeezing increases with the linear gain coefficient for small values of $\eta$ and almost perfect squeezing can be obtained for large values of the linear gain coefficient. Finally our result shows that it is possible to obtain more squeezing occur in cascade than the superposed, but the quadrature variance for $V$ is constant.

\section{References}

[1] Fesseha Kassahun, Fundamentals of Quantum Optics (Lulu Press Inc., North Carolina, 2008).

[2] Marlan O. Scully and M. Suhail Zubairy, Quantum Optics (Cambridge University, 1997).

[3] Mark Fox, Quantum Optics an Introduction, (Oxford University press, 2006).

[4] Misrak Getahun, Three-Level Laser Dynamic with Coherent and Squeezed Light, PhD Dissertation (Addis Ababa University, 2009).

[5] Fesseha Kassahun, Three-Level Laser Dynamics with the Atoms Pumped by Electron bombardment, (Addis Ababa University, 2012).

[6] Assegid Mengistu, MSc Thesis, (Addis Ababa University, 2010).

[7] S. M. Barnett and P. M. Raymore, Methods in Theoretical Quantum Optics, (Oxford, University Press, New York, 1997).

[8] Tewodros Yifrashewa Darge, Coherently Driven Three-Level Laser with Parametric Amplifier, PhD Dissertation (Addis Ababa University, 2010).

[9] M. O. Scully and M. S. Zubairy, Quantum Optics, (Cambridge University Press, 1997).

[10] Wobshet Mekonen a non degenerate three-level laser with cavity modes driven by coherence light MSc Thesis, (Addis Ababa University, 2007).

[11] Sintayehu Tesfa, arxiv: 07082815v1 [quanta- ph. (2007).

[12] Sintayehu Tesfa a No degenerate Three-level Cascade Laser coupled to a Two- mode Squeezed Vacuum Reservoir PhD Dissertation (Addis Ababa University 2008).

[13] Economou, Sophia Spontaneous emission and optical control of spins in quantum dots PhD Dissertation (UNIVERSITY OF CALIFORNIA, 2006).

[14] Beyene Abiti Superposed De gene rate Three-Level Lasers MSc thesis (Addis Ababa University 2011).

[15] Tizazu Maresha Superposition of Squeezed Laser Light Beams PhD Dissertation (Addis Ababa University 2015).

[16] Solomon Getachew TWO-MODE COHERENT AND LASER LIGHT BEAMS MSc thesis (Addis Ababa University June 2011).

[17] Peter Lambropoulos David Petrosyan Fundamentals of Quantum Optics and Quantum Information (Springer). 